\title{
Docking studies, synthesis, characterization, and cytotoxicity activity of new bis-chalcone derivatives
}

\author{
Mohammad Murwih Alidmat ${ }^{1}$, Melati Khairuddean ${ }^{1}{ }^{*}$, Salizawati Muhamad Salhimi ${ }^{2}$, Mohammad Al-Amin ${ }^{2}$
}

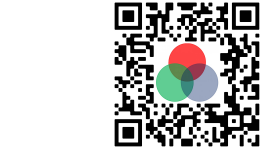

Use your smartphone to scan this QR code and download this article

${ }^{1}$ School of Chemical Sciences, Universiti Sains Malaysia, 11800 Penang, Malaysia

${ }^{2}$ School of Pharmaceutical Sciences, Universiti Sains Malaysia, 11800 Penang, Malaysia

\section{Correspondence}

Melati Khairuddean, School of Chemical Sciences, Universiti Sains Malaysia, 11800 Penang, Malaysia

Email:melati@usm.my

\section{History}

- Received: Dec 29, 2020

- Accepted: Apr 15, 2021

- Published: Apr 29, 2021

DOI : 10.15419/bmrat.v8i4.668

\section{Check for updates}

\section{Copyright}

(c) Biomedpress. This is an openaccess article distributed under the terms of the Creative Commons Attribution 4.0 International license.

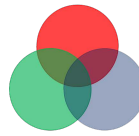

\begin{abstract}
Introduction: Breast cancer is women's most prevalent cancer type. The development of chemotherapy drug resistance and adverse effects is a significant barrier to breast cancer treatment. Recently, the focus of drug discovery has increased toward a valuable structure known as chalcones due to their extensive bioactivity in cancer treatment. Methods: The molecular docking studies were conducted using the Discovery Studio (DSv4.5) and MG. Tools installed in Window 10. The cancer receptor (3ERT) was downloaded from the protein data bank (PDB). All new compounds were characterized by IR, 1H-NMR, 13C-NMR, 2D-NMR, and CHN elemental analysis. Results: $A$ bis-chalcones (1-13) were prepared by the reaction of terephthalaldehyde with 3-acetyl2,5-dichlorothiophene or 3-acetyl-5-chlorothiophene and reaction cyclo ketone derivatives with phenyl aldehyde derivatives in good yields. All the synthesized bis chalcone derivatives have been characterized using ATR-FTIR, NMR (1D and 2D). The cytotoxicity activity of all these chalcone compounds was investigated against breast cancer cell line (MCF-7). The results showed that compound 6 showed more potent activity in inhibiting growth on both types of MCF-7 IC 50 (4.4 \pm 0.10 $\mu \mathrm{M})$ compared to reference tamoxifen $\mathrm{IC}_{50}(17.9 \pm 1.2 \mu \mathrm{M})$. Conclusion: The cytotoxic activity of all the synthesized compounds was evaluated against MCF-7 breast cancer cell lines. Compounds $\mathbf{5}$ and $\mathbf{6}$ were found to have $\mathrm{IC}_{50}$ values of about $20 \mu \mathrm{M}$. These compounds are good candidates to be selected for further studies to develop anticancer drugs. The structure-activity relationships study showed that bis-chalcone compounds with substituted chlorine and flurine atom at the ortho-chlorine and para-flurine position of their aromatic rings exhibited greater cytotoxic activity against MCF-7 cell line compared to other compounds. Moreover, in the cytotoxic activity test towards MCF-7 breast cancer cells, bis-chalcones derivatives from cyclohexane, 1-ethylpiperidin-4one were found to be more potent than bis-chalcone derivatives from acetone, cyclopentanone, 4-(tert-butyl)cyclohexane-1-one, and terephthalaldehyde.
\end{abstract}

Key words: bis-chalcone, cyclo ketone, breast cancer, tamoxifen, terephthalaldehyde

\section{INTRODUCTION}

The prevalence of cancer is rising worldwide, making it the highest leading cause of death in economically developed countries and the second leading cause of death in developing countries. Cancer is considered one of the most intractable diseases because of the innate characteristics of cancer cells to proliferate uncontrollably, avoid apoptosis, invade and metastasize $^{1}$. Despite the advances in chemotherapy, there are no sufficient clinically useful cytotoxic agents that can selectively target cancer cells ${ }^{2}$. Even after recent advances in understanding the biological processes leading to the development of cancer, there is still a need for new and effective agents to help bring this disease under control ${ }^{3}$. Among the currently identified anti-tumor agents, chalcones represent an important class of abundant molecules from edible plants ${ }^{4,5}$. Chalcones are open-chained molecules bearing two aromatic rings linked by a three-carbon enone fragment ${ }^{6,7}$. Many of these molecules exhibit beneficial biological activities, including antiprotozoal $^{8}$, antifungal ${ }^{9}$, anti-inflammatory ${ }^{10}$, antileishmanial $^{11}$, antioxidant ${ }^{12}$, and anticancer ${ }^{10,13,14}$, as well as many other biological activities ${ }^{15}$. Recent studies on the anti-proliferative and tumor-reducing activities of some chalcones have led to an increased interest to investigate chalcone compounds as anticancer agents due to their simple chemical structure, their large number of sub-stituents that can create an enormous number of derivatives, their ease of synthesis, and their wide range of bioactivities ${ }^{5,16}$. Such compounds with anti-tumor properties have prompted us to synthesize a series of new chalcones and to further investigate the anti-proliferative ability of these chalcones against various breast cancer cell lines.

Estrogen receptors $\mathrm{ER} \alpha$ and $\mathrm{ER} \beta$ are significant prognostic markers with different estrogen affinities to 
identify tumors in the breast tissue ${ }^{17}$. ER $\alpha$ serves as an activated site for the ligand, and is essential in the development and progression of dependent hormonal type breast cancer ${ }^{18,19}$. Tamoxifen is an anti-estrogen that blocks the estrogenic signal and binds to the estrogen receptors, thus modifying their activities. Tamoxifen and its active metabolite 4hydroxytamoxifen (4-OHT) have cytotoxic activity against MCF-7 breast cancer cells. However, the efficacy of tamoxifen is limited by the presence of potential resistance. One of the current strategies to understand the drug-receptor relationship in modern drug discovery is through molecular docking. Molecular docking is a computational method that provides information about intermolecular interactions of proteins, nucleic acids, lipids, and ligands. Molecular docking gives the optimized conformation of proteins and ligands, and their relative orientation through their minimized binding energy ${ }^{20}$.

\section{MATERIALS - METHODS}

\section{Chemicals and Solvents}

The chemicals and reagents used in the synthesis and characterization experiments were as follows: ethyl acetate, AR grade (QRëC); n-Hexane, AR grade (QRëC); 4-benzyloxy-3-methoxybenzaldehyde (Sigma-Aldrich, St. Louis, MO, USA); 4chloro-6-florobenzaldehyde (Sigma-Aldrich); 2-chloro-6-florobenzaldehyde (Sigma-Aldrich);

3-acetyl-2,5-dichlorothiophene (Sigma-Aldrich); terephthalaldehyde (Sigma-Aldrich); cyclopentanone (Sigma-Aldrich); cyclohexanone (Sigma-Aldrich); sulphuric acid, 95-97\%, AR grade (QRëC); 2,6dichlorobenzaldehyde (Sigma-Aldrich, USA); 2-acetyl-5-chlorothiophene (Sigma-Aldrich); 4(tert-butyl)cyclohexan-1-one (Sigma-Aldrich); paraffin oil, extra pure (QRëC); 3-benzyloxy-4methoxybenzaldehyde (Sigma-Aldrich); dimethyl sulfoxide-d6 (Sigma-Aldrich); chloroform-d (ARMAR Chemicals, Switzerland); 1-ethylpiperidin4-one (Sigma-Aldrich); potassium hydroxide (SigmaAldrich); sodium hydroxide (Sigma-Aldrich); and TLC silica gel 60 F254 aluminium sheet, $20 \mathrm{~cm}$ $\times 20 \mathrm{~cm}$ (Merck, Germany). All solvents were used without additional purification unless stated otherwise.

\section{Thin Layer Chromatography (TLC)}

TLC was used to monitor reaction progress and to identify products in the mixture. A sample dissolved in solvent was spotted at the bottom of the TLC plate. The plate was then inserted into the eluent of ethyl acetate:n-hexane with a different ratio. The ratio used was $5: 95,10: 90,15: 85,20: 80$ and $25: 75$ of ethyl acetate:hexane. The product was identified by comparing its $\mathrm{Rf}$ value with the $\mathrm{Rf}$ value of the known compound or the starting material under UV light.

\section{Recrystallization}

All compounds were purified by re-crystallization techniques. The slower the rate of cooling, the larger the crystals were that formed. A major disadvantage of re-crystallization is the length of time it takes. Moreover, the proper solvent must be used but this can only be determined by trial and error, based on predictions and observations. The solution must be soluble at high temperatures and insoluble at low temperatures. The advantage of re-crystallization is that when carried out correctly, it is a very effective way of obtaining a pure sample of the product or precipitate.

\section{Fourier Transform Infrared Spectroscopy (FTIR)}

FTIR spectroscopy is a technique to determine the types of functional groups in a compound. The frequency of infrared is measured in wave number, and the samples are scanned in a range from 650 to 4000 $\mathrm{cm}^{-1}$

\section{Nuclear Magnetic Resonance Spectroscopy (NMR)}

NMR spectroscopy is used to determine the molecular structure of a compound for certain atomic nuclei such as $1 \mathrm{H}, 13 \mathrm{C}$, and $31 \mathrm{P}$. The $1 \mathrm{D}$ NMR (1H and 13C) and 2D NMR (COSY, HSQC, HMBC, DEPT90, and DEPT135) were carried out to confirm the structures of the intermediates and final compounds. All the NMR spectra were obtained using Bruker $500 \mathrm{MHz}$ UltrashieldTM spectrometer. About $20 \mathrm{mg}$ of sample was dissolved in a deuterated solvent $\left(\mathrm{CDCl}_{3}-\mathrm{d} 1\right.$ and DMSO-d6) before transfer into an NMR tube.

\section{CHN elemental analysis}

CHN elemental analysis was used to determine the percentage of carbon $(\mathrm{C})$, hydrogen $(\mathrm{H})$, and nitrogen $(\mathrm{N})$ in a sample. This technique involves the combustion of a sample in excess of oxygen. It provides a speedy and inexpensive method to check the purity of a sample. The analysis of the synthesized samples was carried out using the CHN analyzer (Perkin Elmer II 2400 model, Perkin Elmer, Waltham, MA, USA). 


\section{Cytotoxicity Study}

\section{Cell Culture}

Human breast cancer cell line MCF-7 was purchased from ATCC (Rockville, MD, USA). The cells were maintained in Dulbecco's Modified Eagle's medium (DMEM) supplemented with $10 \% \mathrm{Fe}$ tal Bovine Serum (FBS) and incubated at $37{ }^{\circ} \mathrm{C}$ with humidified condition maintained with $5 \% \mathrm{CO}_{2}$. After reaching confluency, cells were sub-cultured using $0.25 \%$ trypsin- EDTA. All aforementioned reagents were purchased from Thermo-Fisher Scientific (Waltham, MA, USA).

\section{Cell Viability Assay}

The cytotoxicity of 30 compounds was examined against MCF-7 cells by using cell viability assay. Briefly, MCF-7 cells were seeded into a 96-well plate at a density of $4 \times 10^{3}$ cells/well and incubated for 24 h. The cells were treated with synthetic compounds, the reference drug tamoxifen, and 0.1\% DMSO as control, and were incubated for $48 \mathrm{~h}$. Following the incubation period, the media with treatments were discarded, and new medium was added to each well to minimize any potential interaction of drugs with the MTT reagent. Then, $20 \mu \mathrm{L}$ of the MTT reagent was added into each well and incubated for $3 \mathrm{~h}$. The medium containing MTT was discarded and $200 \mu \mathrm{L}$ of DMSO was added to each well and incubated. After $15 \mathrm{~min}$, the absorbance was measured by using a plate reader at a wavelength of $570 \mathrm{~nm}$ with a reference wavelength of $630 \mathrm{~nm}$. The $\mathrm{IC}_{50}$ values of the compounds which showed an effect on the viability of the cells at a concentration below $100 \mu \mathrm{M}$ were calculated using five concentrations.

\section{Molecular Docking \\ Protein Preparation}

The X-ray crystal structure of $\mathrm{ER} \alpha$ was downloaded from the RCSB database (PDB ID: 3ERT) ${ }^{21}$. Biovia Discovery Studio Visualizer 16.1 was utilized to remove the heteroatoms and water, and to further prepare the protein.

\section{Ligand Preparation}

Eleven synthesized compounds were used as the ligands while tamoxifen was selected as a control reference in the docking studies. The $2 \mathrm{D}$ chemical structure of the ligands was built using PerkinElmer ChemDraw software 16.0 (Perkin Elmer). Next, the sketched ligands were subjected to energy minimization (MM2 force field) using PerkinElmer Chem3D 16.0 and saved in PDB format.

\section{Docking and Scoring Protocol}

AutoDock 4.2 is a computational software used to prepare the ligands and proteins, and to generate the docking process ${ }^{22}$. A click-by-click protocol was used to enforce this process ${ }^{23}$. Initially, the polar hydrogens and Kollman charges were added to the $\mathrm{ER} \alpha$. Then, the selected ligands were revitalized by Gasteiger charges. The size of the grid box was set to $50 \star 50 \star 50$, and the coordinates were $31.6615,-0.8435$, and 25.1743 (as $x, y$ and $z$, respectively) with a spacing of 0.375 . For the docking parameter, the ER $\alpha$ was defined as rigid while all ligands were flexible. The genetics algorithm run was set to 100 , and Lamarckian genetics was selected to proceed with the docking, while the remaining parameters were kept as default. Docking scores were interpreted using Discovery Studio Visualizer 16.1 and LigandScout 4.3 academic license so that the ionic bonds, hydrogen bonds, and hydrophobic interactions could be easily observed.

\section{RESULTS}

\section{Synthesis}

The general procedure of chalcone preparations (for bis-chalcones 1-13) involved the Claisen-Schmidt condensation of various substituted thiophenes with substituted benzaldehydes in methanol and potassium hydroxide $(\mathrm{KOH})$ as bases. Two types of bischalcone derivatives, 1-3 and 4-13, were synthesized. The reaction conditions involved are summarized in Scheme 1 and Scheme 2, respectively.

\section{General procedure of bis-chalcone deriva- tives, 1-3}

The preparation of Bis-chalcone $\mathbf{1}$ was used as a representative procedure. A mixture of 2-acetyl-5chlorothiophene $(0.02 \mathrm{~mol})$ and terephthalaldehyde $(0.01 \mathrm{~mol})$ in $50 \mathrm{~mL}$ methanol was stirred in the presence of aqueous $\mathrm{KOH}(0.04 \mathrm{~mol}, 4$ equiv) at room temperature for 24 hours. The reaction progress was monitored by TLC. The precipitate formed was filtered and purified by recrystallization from methanol. The reaction is illustrated in Scheme 1.

(2E,2'E)-3,3'-(1",4"-Phenylene)-bis-(1',1(5",', $5^{\prime \prime \prime \prime}-$-chlorothiophen-2"',2'"'-yl) prop-2,2'en-1,1'-one), 1

Yield: (68\%); color: solid yellow; mp: $170-174{ }^{\circ} \mathrm{C}$; molecular weight: 419.34. FT-IR $\left(v, \mathrm{~cm}^{-1}\right): 3067$ (Csp2-H), 3001 and $2936(\mathrm{Csp} 3-\mathrm{H}), 1657(\mathrm{C}=\mathrm{O})$, 1523 ( $\mathrm{C}=\mathrm{C}$ aromatic), 1375 ( $\mathrm{C}=\mathrm{C}$ alkenyl), and 614 (C-Cl). ${ }^{1} \mathrm{H}-\mathrm{NMR}\left(500 \mathrm{MHz}, \mathrm{CDCl}_{3}\right) \mathrm{d}, \mathrm{ppm}: 7.85$ (2H, d, J=15.5 Hz, H-2,2'), 7.70 (4H, s, H-2",3",5",6”), 


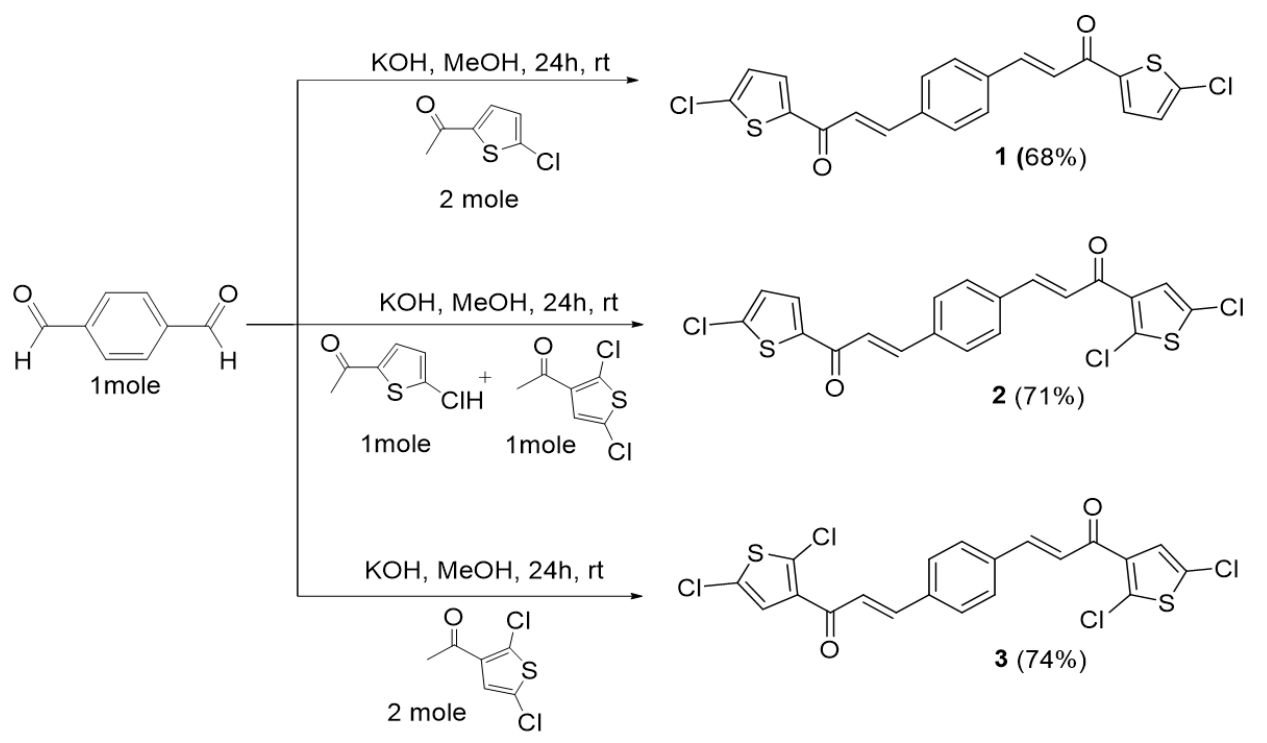

Scheme 1: Synthesis of chalcones 1-3.

$7.69(2 \mathrm{H}, \mathrm{d}, J=4.0, \mathrm{H}-3$ ") $7.39(2 \mathrm{H}, \mathrm{d}, J=15.5 \mathrm{~Hz}$, $\left.\mathrm{H}-3,3^{\prime}\right)$, and $7.05\left(2 \mathrm{H}, \mathrm{d}, 1 \mathrm{H}, J=4.0 \mathrm{~Hz}, \mathrm{H}-4^{\prime \prime}\right) .{ }^{13} \mathrm{C}$ NMR (125 MHz, $\mathrm{CDCl}_{3}$ ) d, ppm: 190.0 (C-1,1'), 144.0 (C-2,2'), 142.6 (C-3"'), 140.1 (C-2"'), 131.8 (C5”), 129.1 (C-2",3",5",6"), 129.0 (C-1",4”), 128.8 (C4"'), and 127.7 (C-3,3'). CHN Elemental analysis: calculated for $\mathrm{C}_{20} \mathrm{H}_{12} \mathrm{O}_{2} \mathrm{Cl}_{2} \mathrm{~S}_{2}$ : C, 57.28; $\mathrm{H}, 2.88$; found: C, 57.31; H, 2.76 (Figure 1).

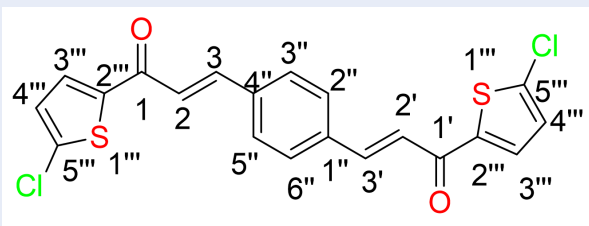

Figure 1: (2E,2'E)-3,3'-(1",4"--Phenylene)-bis$\left(1,1-\left(5^{\prime \prime \prime}, 5^{\prime \prime \prime \prime}-\right.\right.$ chlorothiophen-2"',2"'"-yl)prop-2,2'en-1,1'-one), 1

(2E,2'E)-3,3'-(1",4"-phenylene)-bis-(1,1'(2",'5"'-dichlorothiophen-3"'-yl) prop-2,2'en-1,1'-one), 2

A mixture of 2-acetyl-5-chlorothiophene $(0.01 \mathrm{~mol})$, 3-acetyl-2,5-dichlorothiophene $(0.01 \mathrm{~mol})$, aqueous $\mathrm{KOH}$ (0.04 mol, 4 equiv) and terephthalaldehyde $(0.01 \mathrm{~mol})$ in $50 \mathrm{~mL}$ methanol was stirred at room temperature for 24 hours.

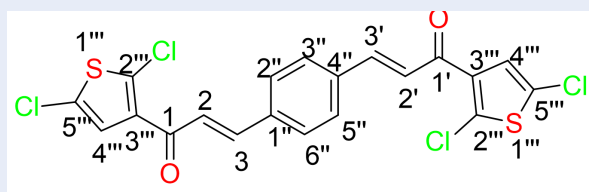

Figure 2: $\quad\left(2 E, 2^{\prime} E\right)-3,3^{\prime}-\left(1^{\prime \prime}, 4^{\prime \prime}\right.$-phenylene)-bis(1,1'-(2'",5"'-dichlorothiophen-3"'-yl) prop-2,2'en-1,1'-one), 2

Yield: (71\%); color: solid yellow; mp: $163-167{ }^{\circ} \mathrm{C}$; and molecular weight: 488.23. FT-IR $\left(v, \mathrm{~cm}^{-1}\right)$ : $3080\left(\mathrm{C}_{s p}{ }^{2}-\mathrm{H}\right.$ str.), $2972\left(\mathrm{C}_{s p}{ }^{3}-\mathrm{H}\right.$ str.), 1650 (C=O str.), 1588 ( $\mathrm{C}=\mathrm{C}$ aromatic str.), 1498 ( $\mathrm{C}=\mathrm{C}$ alkenyl str.), and $719(\mathrm{C}-\mathrm{Cl}) .{ }^{1} \mathrm{H}-\mathrm{NMR}\left(500 \mathrm{MHz}, \mathrm{CDCl}_{3}\right)$ d; ppm: $7.71(\mathrm{~d}, 2 \mathrm{H}, J=15.6 \mathrm{~Hz}, \mathrm{C}=\mathrm{CH}), 7.30(\mathrm{~d}, 2 \mathrm{H}$, $J=15.6 \mathrm{~Hz}, \mathrm{CO}=\mathrm{CH}), 7.64$ (s, 4H, Ar-H), 7.09 (s, 2H, thiophene-H). 13C NMR (125 MHz, $\left.\mathrm{CDCl}_{3}\right)$, ppm: 186.1 (C-1,1'), 147.8 (C-2, 2'), 142.4 (C-3"'), 136.8 (C-2”), 136.5 (C-5”), 135.5 (C-2”,3”,5”,6”), 130.2 (C1",4"), 125.8 (C-4"'), and 116.0 (C-3,3'). CHN Elemental analysis: calculated for $\mathrm{C}_{20} \mathrm{H}_{10} \mathrm{O}_{2} \mathrm{Cl}_{4} \mathrm{~S}_{2}$ : C, 49.20; H, 2.06; found: C, 49.31; H, 2.10.C, 49.20; H, 2.06; found: C, 49.31; H, 2.10 (Figure 2). 
(E)-1-(5'-chlorothiophen-2'-yl)-3"'--(4"-((E)$3^{\prime \prime \prime}-\left(2^{\prime \prime \prime \prime}, 5^{\prime \prime \prime \prime}-\right.$ dichlorothiophen-3"''-yl)-3"'oxoprop-1"'-en-1"'-yl) phenyl) prop-2-en-1one, 3

A mixture of 3-acetyl-2,5-dichlorothiophene (0.02 $\mathrm{mol})$, aqueous $\mathrm{KOH}$ (0.04 mol, 4 equiv) and terephthalaldehyde $(0.01 \mathrm{~mol})$ in $50 \mathrm{~mL}$ methanol was stirred at room temperature for 24 hours.

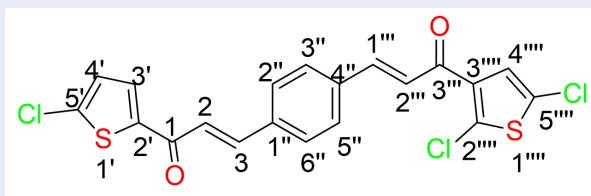

Figure 3: (E)-1-(5'-chlorothiophen-2'-yl)-3"'-(4"((E)-3"'-(2'"',5"'"-dichlorothiophen-3"'"-yl)-3"'oxoprop-1"'-en-1"'-yl) phenyl) prop-2-en-1-one, 3

Yield: (77\%); color: solid yellow; mp: $190-195$ ${ }^{\circ} \mathrm{C}$; and molecular weight: 453.8. FT-IR $\left(v, \mathrm{~cm}^{-1}\right)$. $3034\left(\mathrm{C}_{s p}{ }^{2}-\mathrm{H}\right.$ str.), $2972\left(\mathrm{C}_{s p}{ }^{3}-\mathrm{H}\right.$ str. $), 1650(\mathrm{C}=\mathrm{O}$ str.), 1588 ( $\mathrm{C}=\mathrm{C}$ aromatic str.), 1508 ( $\mathrm{C}=\mathrm{C}$ alkenyl str.), $716(\mathrm{C}-\mathrm{Cl}) .{ }^{1} \mathrm{H}-\mathrm{NMR}\left(500 \mathrm{MHz}, \mathrm{CDCl}_{3}\right) \mathrm{d}$, ppm: $7.84(1 \mathrm{H}, \mathrm{d}, J=15.5 \mathrm{~Hz}, \mathrm{H}-2$ '”), $7.76(1 \mathrm{H}, J=16$ Hz, H-1"'), 7.68 (4H, d, J=8.0 Hz, H-2",3",5",6"), 7.68 $(1 \mathrm{H}, \mathrm{d}, J=4.1 \mathrm{~Hz}, \mathrm{H}-4$ ') $7.45(1 \mathrm{H}, \mathrm{d}, 1 \mathrm{H}, J=16.0 \mathrm{~Hz}$, H-2), 7.38 (1H, d, J=15.5 Hz, H-3), 7.24 (1H, s, H$1^{\prime \prime \prime \prime), ~ a n d ~} 7.04\left(1 \mathrm{H}, \mathrm{d}, J=4.1 \mathrm{~Hz}, \mathrm{H}-4{ }^{\prime}\right) .{ }^{13} \mathrm{C} \mathrm{NMR}$ $\left(125 \mathrm{MHz}, \mathrm{CDCl}_{3}\right) \mathrm{d}$, ppm: 183.6 (C-3"'), 180.7 (C1), 144.1 (C-2'), 144.0 (C-1'”), 143.9 (C-3), 143.1 (C2'”'), 140.2 (C-3'), 137.7 (C-4'), 136.7 (C-1",4”), 131.4 (C-4'), 129.2 (C-2",3”), 129.1 (C-5",6”), 127.8 (C-4"'), 127.2 (C-5'”), 127.1 (C-3"'), 124.8 (C-2"'), 124.7 (C2), and $121.6\left(\mathrm{C}-4{ }^{\prime}\right)$. CHN elemental analysis: calculated for $\mathrm{C}_{20} \mathrm{H}_{11} \mathrm{O}_{2} \mathrm{Cl}_{3} \mathrm{~S}_{2}$ : $\mathrm{C}, 52.94 ; \mathrm{H}, 2.44$; found: $\mathrm{C}$, 52.88; H, 2.40.

General procedure of bis-chalcone derivatives from cycloketones with halogen substituents, 4-7

Bis-chalcone 4 preparation was used as a representative procedure. A mixture of cyclopentanone (0.01 $\mathrm{mol})$ and 2-chloro-4-fluorobenzaldehyde (0.02 mol) in $50 \mathrm{~mL}$ methanol was stirred in the presence of aqueous $\mathrm{KOH}$ (0.04 mol, 4 equiv) at room temperature for 24 hours. The reaction progress was monitored by TLC. The precipitate formed was filtered and purified by re-crystallization from methanol. The reaction is illustrated in Scheme 2.
(2E,5E)-2,5-bis-(2-chloro-4fluorobenzylidene) cyclopentanone, 4

Yield: (85\%); color: solid yellow; mp: $217-222{ }^{\circ} \mathrm{C}$; and molecular weight: 365.20 . FT-IR $\left(v, \mathrm{~cm}^{-1}\right): 3083$ ( $\mathrm{C}_{s p}{ }^{2}-\mathrm{H}$ str.), 2926 ( $\mathrm{C}_{s p}{ }^{3}-\mathrm{H}$ str.), 1686 (C=O str.), 1595 (C=C aromatic str.), 1480 ( $\mathrm{C}=\mathrm{C}$ alkenyl),1041 (C-F), and 808 (C-Cl). ${ }^{1} \mathrm{H}-\mathrm{NMR}\left(500 \mathrm{MHz}, \mathrm{CDCl}_{3}\right.$ ) d, ppm: 7.88 (br s, $2 \mathrm{H}, \mathrm{C}=\mathrm{CH}), 7.25(2 \mathrm{H}, \mathrm{dd}, J=2.6$, $8.5 \mathrm{~Hz}, \mathrm{H}-5$ '), 7.04-7.06 (2H, m, H-3"), and 3.00 (s, cyclic pentane-H, 4H). 13C NMR (125 MHz, CDCl3), ppm: 195.2 (C-1), 163.6 (C-4'), 138.8 (C-1"), 137.2 (C-1'), 131.2 (C-2"), 130.2 (C-6"), 129.1 (C-3"), 117.8 (C-5”), 114.3 (C-2,5), and 26.5 (C-3,4) (Figure 4).

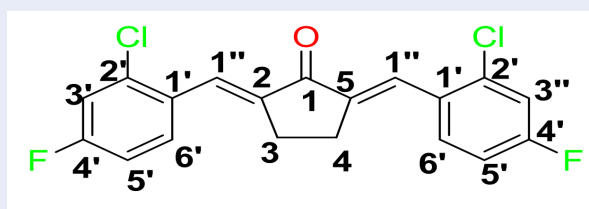

Figure 4: $\quad(2 E, 5 E)-2,5-b i s-(2-c h l o r o-4-$ fluorobenzylidene) cyclopentanone, 4.

\section{(2E,6E)-2,6-bis-(2-chloro-4-}

\section{fluorobenzylidene) cyclohexanone, 5}

A mixture of cyclohexanone $(0.01 \mathrm{~mol})$ and 2-chloro4-fluorobenzaldehyde $(0.02 \mathrm{~mol})$ in $50 \mathrm{~mL}$ methanol was stirred in the presence of aqueous $\mathrm{KOH}(0.04 \mathrm{~mol}$, 4 equiv) at room temperature for 24 hours.

Yield: (79\%); color: solid yellow; mp: $148-153{ }^{\circ} \mathrm{C}$; and molecular weight: 379.23 . FT-IR $\left(v, \mathrm{~cm}^{-1}\right): 3113$ ( $\mathrm{C}_{s p}{ }^{2}-\mathrm{H}$ str.), 2975 ( $\mathrm{C}_{s p}{ }^{3}-\mathrm{H}$ str.), 1664 (C=O str.), 1598 (C=C aromatic str.), 1486 ( $\mathrm{C}=\mathrm{C}$ alkenyl), 1047 (C-F), and 736 (C-Cl). ${ }^{1} \mathrm{H}-\mathrm{NMR}\left(500 \mathrm{MHz}, \mathrm{CDCl}_{3}\right.$ ) d, ppm: 7.86 (2H,s, H-1"), 7.22 - 7.34 (4H, m, H-3', H-6'), 7.03 (2H, dd, J=3.5, $8.75 \mathrm{~Hz}, \mathrm{H}-5$ ') $2.77(4 \mathrm{H}$, $\mathrm{t}, J=5.2 \mathrm{~Hz}, \mathrm{H}-3,5)$, and $1.76-1.73(2 \mathrm{H}, \mathrm{m}, \mathrm{H}-4) .{ }^{13} \mathrm{C}$ NMR (125 MHz, $\mathrm{CDCl}_{3}$ ) d, ppm: 189.5 (C-1), 163.3 (C-4'), 137.7 (C-1'), 136.1 (C-2), 133.2 (C-2'), 131.4 (C-6'), 130.5 (C-1'), 117.2 (C-3'), 113.7 (C-5'), 28.37 (C-3), and 23.14 (C-4) (Figure 5).

\section{3,5-bis-((E)-2-chloro-4-fluorobenzylidene)- 1-ethylpiperidin-4-one, 6}

A mixture of 1-ethylpiperidin-4-one $(0.01 \mathrm{~mol})$ and 2-chloro-4-fluorobenzaldehyde $(0.02 \mathrm{~mol})$ in $50 \mathrm{~mL}$ methanol was stirred in the presence of aqueous $\mathrm{KOH}$ ( $0.04 \mathrm{~mol}, 4$ equiv) at room temperature for 24 hours. Yield: (84\%); color: solid yellow; mp: $147-153{ }^{\circ} \mathrm{C}$; and molecular weight: 407.07. FT-IR $\left(v, \mathrm{~cm}^{-1}\right): 3066$ ( $\mathrm{C}_{s p}{ }^{2}-\mathrm{H}$ str.), 2974 and $2938\left(\mathrm{C}_{s p}{ }^{3}-\mathrm{H}\right.$ asymmetrical 


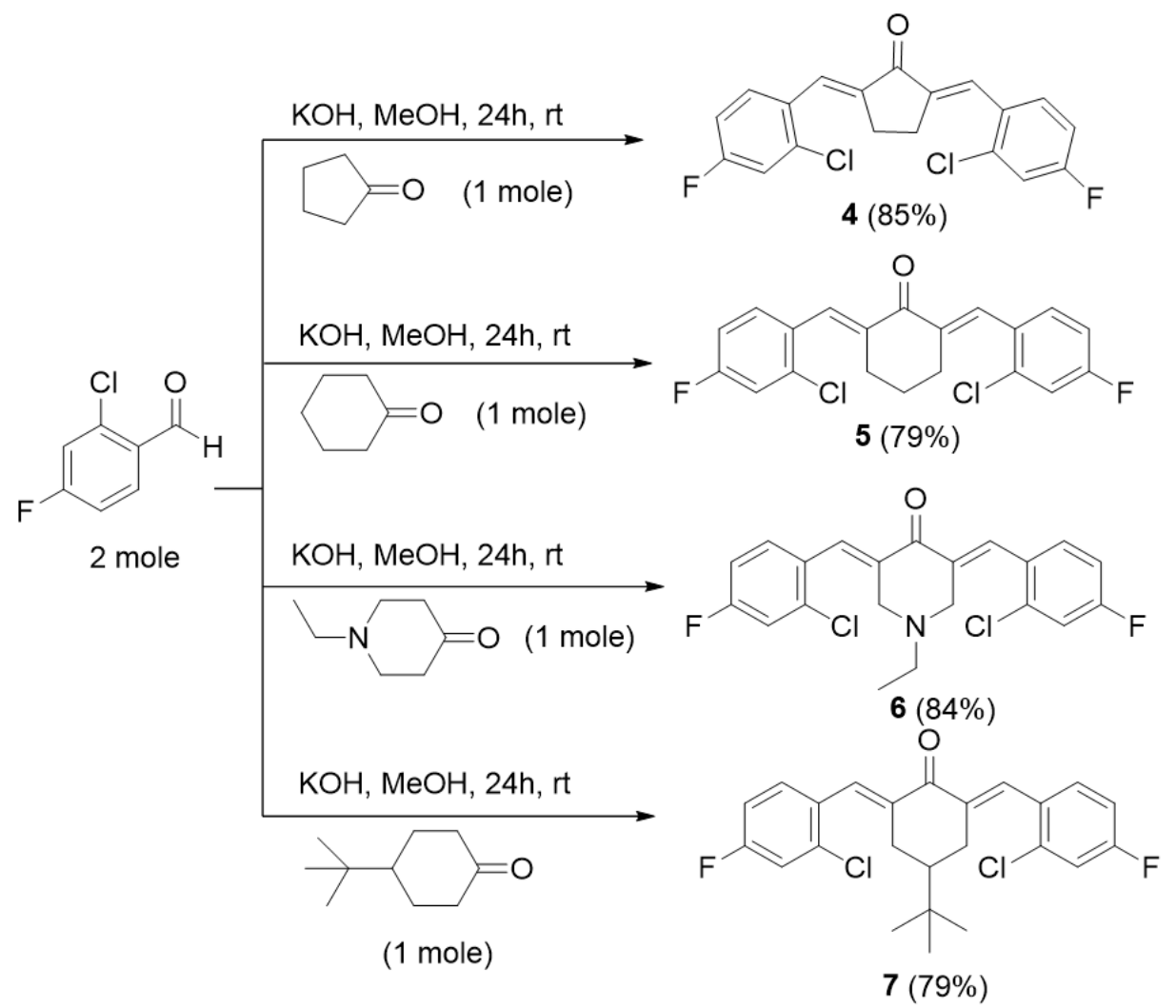

Scheme 2: Synthesis of bis-chalcone with halogen substituents, 4-7.

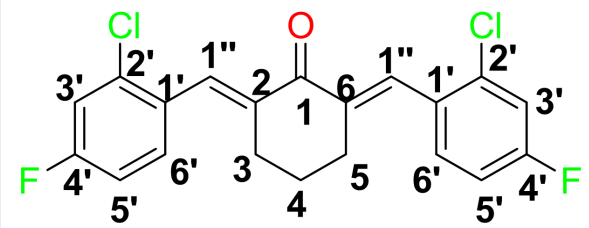

Figure $\quad 5: \quad$ (2E,6E)-2,6-bis-(2-chloro-4fluorobenzylidene) cyclohexanone, 5 .

and symmetrical stretching, respectively), $1679(\mathrm{C}=\mathrm{O}$ str.), 1601 ( $\mathrm{C}=\mathrm{C}$ aromatic str.), 1488 ( $\mathrm{C}=\mathrm{C}$ alkenyl), $1217(\mathrm{C}-\mathrm{N}), 1045$ (C-F), and 687 (C-Cl). ${ }^{1} \mathrm{H}-\mathrm{NMR}$ (500 MHz, $\mathrm{CDCl}_{3}$ ) d, ppm: 1.07 (t, J=7.0 Hz, H-2", $3 \mathrm{H}), 2.55$ (d, J=5.0 Hz, H-1", $2 \mathrm{H}), 3.66\left(\mathrm{~s}, \mathrm{CH}_{3}, 6 \mathrm{H}\right)$, 3.72 (s, H-2, H-6, 4H), 3.85 (s, $\left.\mathrm{CH}_{2}, 4 \mathrm{H}\right), 6.84-8.02$ (m, H-aromatic, $6 \mathrm{H})$, and $7.94(\mathrm{~s}, \mathrm{CH}, 2 \mathrm{H}) \cdot{ }^{13} \mathrm{C} \mathrm{NMR}$ $\left(125 \mathrm{MHz}, \mathrm{CDCl}_{3}\right)$ d, ppm: $12.2(\mathrm{C}-2$ "), 50.8 (C-1"), 53.8 (C-2,6), 114.0 (C-3'), 129.7 (C-3'), 131.4 (C-1'), 133.1 (C-6'), 134.3 (C-2'), $136.3(\mathrm{C}-3,5), 161.4(\mathrm{CH})$, $163.5\left(\mathrm{C}-4^{\prime}\right)$, and 186.4 (C-4).<smiles>CC(C)N1CC(=Cc2ccc(F)cc2Cl)C(=O)C1=Cc1ccc(F)cc1Cl</smiles>

Figure $\quad 6: \quad 3,5-b i s-((E)-2-c h l o r o-4-$ fluorobenzylidene)-1-ethylpiperidin-4-one,

6.

4-(Tert-butyl)-2,6-bis-((E)-2-chloro-4fluorobenzylidene) cyclohexan-1-one, 7

A mixture of 4-(tert-butyl) cyclohexan-1-one (0.01 $\mathrm{mol})$ and 2-chloro-4-fluorobenzaldehyde $(0.02 \mathrm{~mol})$ in $50 \mathrm{~mL}$ methanol was stirred in the presence of aqueous $\mathrm{KOH}$ ( $0.04 \mathrm{~mol}, 4$ equiv) at room temperature for 24 hours. 
Yield: (79\%); color: solid yellow, mp: $150-155^{\circ} \mathrm{C}$; and molecular weight: 435.33 . FT-IR $\left(v, \mathrm{~cm}^{-1}\right): 3032$ ( $\mathrm{C}_{s p}{ }^{2}-\mathrm{H}$ str.), 2955 ( $\mathrm{C}_{s p}{ }^{3}-\mathrm{H}$ str.), 1737 (C=O str.), 1595 ( $\mathrm{C}=\mathrm{C}$ aromatic str.), 1514 ( $\mathrm{C}=\mathrm{C}$ alkenyl), 1010 (C-F), and $700(\mathrm{C}-\mathrm{Cl}) .{ }^{1} \mathrm{H}-\mathrm{NMR}(500 \mathrm{MHz}, \mathrm{DMSO}-$ $\mathrm{d}_{6}$ ) d, ppm: 0.80 (s, H-2",3"',4"', 9H), 2.23 (t, J=13.5 $\mathrm{Hz}, \mathrm{H}-1,1 \mathrm{H}), 2.87$ (d, J=15.5 Hz, H-2, H-6, 4H), 6.76$7.82(\mathrm{~m}, \mathrm{H}$-aromatic, $6 \mathrm{H})$, and $7.74(\mathrm{~s}, \mathrm{CH}, 2 \mathrm{H}) .{ }^{13} \mathrm{C}$ NMR (125 MHz, $\mathrm{CDCl}_{3}$ ) d, ppm: 27.1 (C-2",3",4"), 29.3(C-2, 6), 32.6 (C-1"), 55.6 (C-1), 117.2 (C-5'), 117.4 (C-3'), 130.5 (C-1'), 130.5 (C-2'), 131.1 (C-6'), $131.3(\mathrm{C}-3,5), 131.3(\mathrm{CH}), 160.1\left(\mathrm{C}-4^{\prime}\right)$, and $189.6(\mathrm{C}-$ 4) (Figure 7).

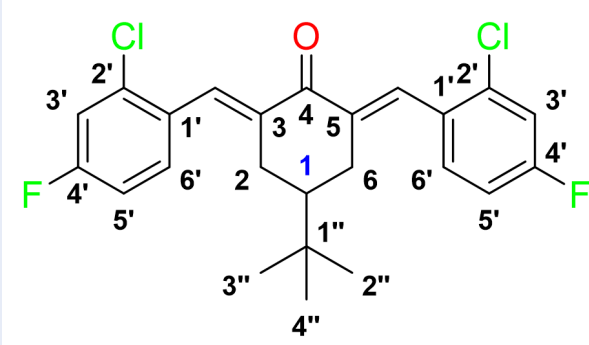

Figure 7: 4-(Tert-butyl)-2,6-bis-((E)-2-chloro-4fluorobenzylidene) cyclohexan-1-one, 7.

General procedure of bis-chalcone derivatives from cycloketones with methoxy and benzyloxy substituents, 8-11

Bis-chalcone 8 was used as a representative procedure. A mixture of acetone $(0.01 \mathrm{~mol})$ and 3 (benzyloxy)-4-methoxybenzaldehyde $(0.02 \mathrm{~mol})$ in $50 \mathrm{~mL}$ methanol was stirred in the presence of aqueous $\mathrm{KOH}$ ( $0.04 \mathrm{~mol}, 4$ equiv) at room temperature for 24 hours. The reaction progress was monitored by TLC. The precipitate formed was filtered and purified by re-crystallization from methanol.

\section{(1E,4E)-1,5-bis-(3-(benzyloxy)-4-}

methoxyphenyl) penta-1,4-dien-3-one, 8

Yield: (78\%); color: solid yellow; mp: $185-190$ ${ }^{\circ} \mathrm{C}$; and molecular weight: 506.59 . FT-IR $\left(v, \mathrm{~cm}^{-1}\right)$ : $3031\left(\mathrm{C}_{s p}{ }^{2}-\mathrm{H}\right.$ str.), 2972 and $2838\left(\mathrm{C}_{s p}{ }^{3}-\mathrm{H}\right.$ asymmetrical and symmetrical stretchings, respectively), 1745 (C=O str.), 1584 ( $\mathrm{C}=\mathrm{C}$ aromatic str.), 1512 (C=C alkenyl str.), and 1137 (C-O str.). ${ }^{1} \mathrm{H}-\mathrm{NMR}(500$ $\left.\mathrm{MHz}, \mathrm{CDCl}_{3}\right)$ d, ppm: $3.82\left(\mathrm{~s}, \mathrm{CH}_{3}, 6 \mathrm{H}\right), 5.17$ (s, $\left.\mathrm{CH}_{2}, 4 \mathrm{H}\right), 7.06$ (d, $\left.J=8.0 \mathrm{~Hz}, \mathrm{H}-5,2 \mathrm{H}\right), 7.22$ (d, $J=16.0$,
$\mathrm{H}-1,5), 7.35-7.54$ (m, H-aromatic, 16H), and 7.70 (d, $J=15.6 \mathrm{~Hz}, \mathrm{H}-2,5,2 \mathrm{H}) .{ }^{13} \mathrm{C}-\mathrm{NMR}(125 \mathrm{MHz}$, DMSO$\mathrm{d}_{6}$ ) d, ppm: 56.1 (CH3), 70.5 (CH2), 112.4 (C-2',5'), 112.7 (C-2,4), 124.0 (C-6'), 124.2 (C-2",6”), 127.9 (C1'), 128.4 (C-4"), 128.4 (C-3",5”), 128.9 (C-1"), 137.3 (C-5), 143.0 (C-1), 148.4 (C-4'), 151.8 (C-3'), and 188.6 (C-3) (Figure 8).

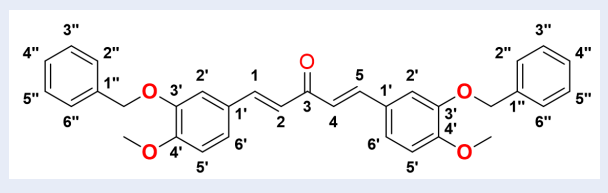

Figure 8: (1E,4E)-1,5-bis-(3-(benzyloxy)-4methoxyphenyl) penta-1,4-dien-3-one, 8.

\section{2,5-bis-((E)-3-(benzyloxy)-4- methoxybenzylidene) cyclopentan-1-one, 9}

A mixture of cyclopentanone $(0.01 \mathrm{~mol})$ and 3(benzyloxy)-4-methoxybenzaldehyde $(0.02 \mathrm{~mol})$ in $50 \mathrm{~mL}$ methanol was stirred in the presence of aqueous $\mathrm{KOH}$ (0.04 mol, 4 equiv) at room temperature for 24 hours.

Yield: (75\%); color: solid yellow; mp: $177-182$ ${ }^{\circ} \mathrm{C}$; and molecular weight: 532.63 . FT-IR $\left(v, \mathrm{~cm}^{-1}\right)$ : $3028\left(\mathrm{C}_{s p}{ }^{2}-\mathrm{H}\right.$ str.), 2955 and $2838\left(\mathrm{C}_{s p}{ }^{3}-\mathrm{H}\right.$ asymmetrical and symmetrical stretchings, respectively), 1742 ( $\mathrm{C}=\mathrm{O}$ str.), 1599 ( $\mathrm{C}=\mathrm{C}$ aromatic str.), 1508 ( $\mathrm{C}=\mathrm{C}$ alkenyl str.), and 1144 (C-O str.). ${ }^{1} \mathrm{H}-\mathrm{NMR}$ $\left(500 \mathrm{MHz}, \mathrm{CDCl}_{3}\right) \mathrm{d}$, ppm: 2.86 (s, H-3,4, $4 \mathrm{H}$ ), $3.96\left(\mathrm{~s}, \mathrm{CH}_{3}, 3 \mathrm{H}\right), 5.23\left(\mathrm{~s}, \mathrm{CH}_{2}, 4 \mathrm{H}\right), 6.95-7.48(\mathrm{~m}$, $\mathrm{H}$-aromatic, $16 \mathrm{H}), 7.12(\mathrm{~s}, \mathrm{CH}, 2 \mathrm{H}) .{ }^{13} \mathrm{C}-\mathrm{NMR}(125$ $\left.\mathrm{MHz}, \mathrm{CDCl}_{3}\right) \mathrm{d}$, ppm: $26.2(\mathrm{C}-3,4), 56.0(\mathrm{CH} 3), 71.1$ (CH2), 111.5 (C-5'), 116.1 (C-6'), 125.3 (C-2'), 127.1 (C-1'), 128.0 (C2",6”), 128.6 (C-4"), 128.8 (C-2",5”), $133.5(\mathrm{CH}), 135.4$ (C-1"), 136.8 (C-2,5), 174.9 (C-4'), 150.9 (C-3'), and 196.0 (C-1) (Figure 9).

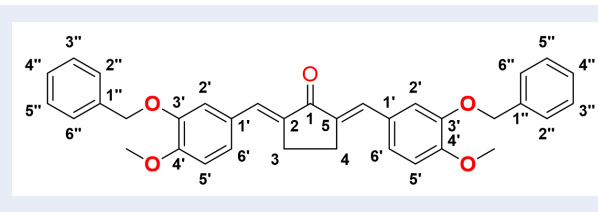

Figure 9: 2,5-bis-((E)-3-(benzyloxy)-4methoxybenzylidene) cyclopentan-1-one, 9. 


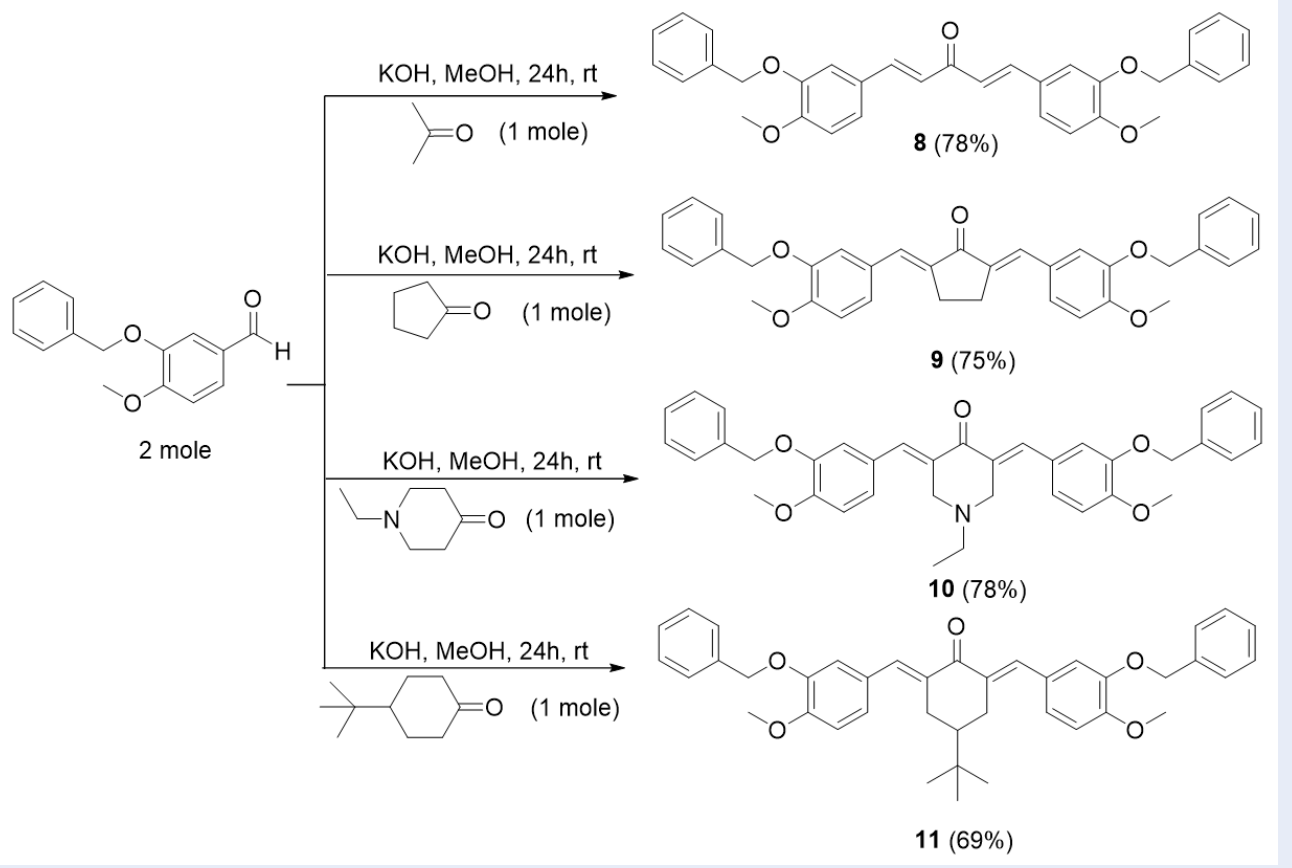

Scheme 3: Synthesis of bis-chalcones with methoxy and benzyloxy substituents, 8-11.

\section{3,5-bis-((E)-3-(benzyloxy)-4- methoxybenzylidene)-1-ethylpiperidin- 4-one, 10}

Yield: (79\%); color: solid yellow; mp: $196-199$ ${ }^{\circ} \mathrm{C}$; and molecular weight: 575.69 . FT-IR $\left(v, \mathrm{~cm}^{-1}\right)$ : 3008 ( $\mathrm{C}_{s p}{ }^{2}-\mathrm{H}$ str.), 2955 and $2841\left(\mathrm{C}_{s p}{ }^{3}-\mathrm{H}\right.$ asymmetrical and symmetrical stretchings, respectively), 1673 ( $\mathrm{C}=\mathrm{O}$ str.), 1605 ( $\mathrm{C}=\mathrm{C}$ aromatic str.), 1486 ( $\mathrm{C}=\mathrm{C}$ alkenyl str.), 1231 (C-N str.), and 1047 (C-O str.). ${ }^{1} \mathrm{H}-\mathrm{NMR}$ (500 MHz, $\mathrm{CDCl}_{3}$ ) d, ppm: 1.01 (t, $J=7.0$ $\mathrm{Hz}, \mathrm{H}-2$ "', 3H), 2.49 (d, J=7.0 Hz, H-1"', 2H), 3.65 (s, $\mathrm{H}-2,6,4 \mathrm{H}), 3.96\left(\mathrm{~s}, \mathrm{CH}_{3}, 6 \mathrm{H}\right), 5.22\left(\mathrm{~s}, \mathrm{CH}_{2}, 4 \mathrm{H}\right)$, 6.93-7.48 (m, H-aromatic, $16 \mathrm{H})$, and 7.69 (s, CH, 2H). ${ }^{13} \mathrm{C} \mathrm{NMR}\left(125 \mathrm{MHz}, \mathrm{CDCl}_{3}\right)$ d, ppm: 12.5 (C-2”), 51.3 (C-1"'), 54.3 (C-2,6), 56.0 (CH3), $71.1(\mathrm{CH} 2)$, 111.4 (C-2'), 116.2 (C-5'), 124.7 (C-6'), 127.0 (2”,6”), 127.5 (C-1'), 128.0 (C-4"), 128.1 (C-3",5"), 128.4 (C1"), 128.7 (C-3,5), $131.6(\mathrm{CH}), 136.2$ (C-4'), 136.8 (C3'), and $187.2(\mathrm{C}-4)$.

\section{2,6-bis-((E)-3-(benzyloxy)-4-} methoxybenzylidene)-4-(tertbutyl)cyclohexan-1-one, 11

Yield: (69\%)l color: solid yellow; mp: $149-154$ ${ }^{\circ} \mathrm{C}$; and molecular weight: 602.76 . FT-IR $\left(v, \mathrm{~cm}^{-1}\right)$ : $3033\left(\mathrm{C}_{s p}{ }^{2}-\mathrm{H}\right.$ str.), 2961 and $2838\left(\mathrm{C}_{s p}{ }^{3}-\mathrm{H}\right.$ asymmetrical and symmetrical stretchings, respectively),

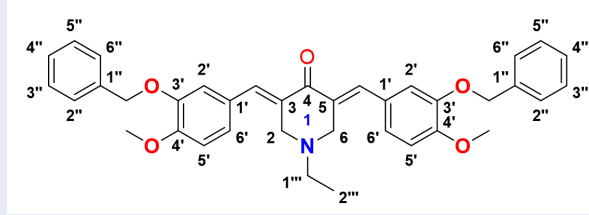

Figure 10: 3,5-bis-((E)-3-(benzyloxy)-4methoxybenzylidene)-1-ethylpiperidin-4-one, 10.

1656 ( $\mathrm{C}=\mathrm{O}$ str.), 1598 ( $\mathrm{C}=\mathrm{C}$ aromatic str.), 1508 ( $\mathrm{C}=\mathrm{C}$ alkenyl str.), and 1140 (C-O str.). ${ }^{1} \mathrm{H}-\mathrm{NMR}(500$ MHz, DMSO-d 6 ) d, ppm: 0.93 (s, H-2", 9H), 1.45 (t, $J=12.5 \mathrm{~Hz}, \mathrm{H}-4,1 \mathrm{H}), 3.1(\mathrm{~d}, J=15.0 \mathrm{~Hz}, \mathrm{H}-3,5,4 \mathrm{H})$, $3.95\left(\mathrm{~s}, \mathrm{CH}_{3}, 6 \mathrm{H}\right), 5.20\left(\mathrm{~s}, \mathrm{CH}_{2}, 4 \mathrm{H}\right), 6.95-7.47(\mathrm{~m}, \mathrm{H}-$ aromatic, $16 \mathrm{H})$, and $7.66\left(\mathrm{~s}, \mathrm{CH}_{2}, \mathrm{H}-5,2 \mathrm{H}\right) .{ }^{13} \mathrm{CNMR}$ (125 MHz, DMSO-d 6 ) d, ppm: 27.4 (C-2"”), 29.4 (C3,5), 32.4 (C-1"'), 44.2 (C-4), 56.0 (CH3), 71.1 (CH2), 111.4 (C-2'), 116.2 (C-5'), 124.5 (C-6’), 127.1 (C-2”, 6”), 127.9 (C-1'), 128.6 (C-4”), 128.9 (C-3",5”), 134.3 (CH), 136.6 (C-1"), 136.8 (C-2,6), 147.8 (C-4'), 150.2 (C-3'), and 190.3 (C-1) (Figure 11). 


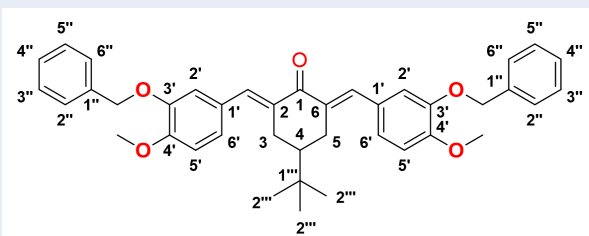

Figure 11: 2,6-bis-((E)-3-(benzyloxy)-4methoxybenzylidene)-4-(tert-butyl)cyclohexan1-one, 11.

General procedure of bis-chalcone derivatives from cycloketones with methoxy and benzyloxy substituents, 12-13

Bis-chalcone 12 preparation was used as a representative procedure. A mixture of cycloketone (0.01 mol) and 3-(benzyloxy)-4-methoxybenzaldehyde $(0.02 \mathrm{~mol})$ in $50 \mathrm{~mL}$ methanol was stirred in the presence of aqueous $\mathrm{KOH}$ ( $0.04 \mathrm{~mol}, 4$ equiv) at room temperature for 24 hours. The reaction progress was monitored by TLC. The precipitate formed was filtered and purified by re-crystallization from methanol (Scheme 4).

\section{3,5-bis-((E)-4-(benzyloxy)-3-} methoxybenzylidene)-1-ethylpiperidin4-one, 12

Yield: (78\%); color: solid yellow; mp: $190-195$ ${ }^{\circ} \mathrm{C}$; and molecular weight: 575.69 . FT-IR $\left(v, \mathrm{~cm}^{-1}\right)$ : FT-IR (v, cm-1): 3032 and 2967 (Csp2-H), 2932 and $2864(\mathrm{Csp} 3-\mathrm{H}), 1669(\mathrm{C}=\mathrm{O}), 1595(\mathrm{C}=\mathrm{C}$ aromatic), $1511(\mathrm{C}=\mathrm{C}$ alkenyl), $1252(\mathrm{C}-\mathrm{N})$, and $1139(\mathrm{C}-\mathrm{O})$. ${ }^{1} \mathrm{H}-\mathrm{NMR}\left(500 \mathrm{MHz}, \mathrm{DMSO}-\mathrm{d}_{6}\right)$ d, ppm: 0.99 (t, $J=7.0 \mathrm{~Hz}, \mathrm{H}-2$ '”, 3H), 2.59 (d, J=7.0 Hz, H-1"', 2H), 3.81 (s, H-2, 6, 4H), 3.82 (s, $\left.\mathrm{CH}_{3}, 6 \mathrm{H}\right), 5.15$ (s, $\mathrm{CH}_{2}$, $4 \mathrm{H}), 7.05-7.47$ (m, H-aromatic, $16 \mathrm{H})$, and $7.58(\mathrm{~s}, \mathrm{CH}$, 2H). ${ }^{13} \mathrm{C}$ NMR (125 MHz, DMSO-d 6 ) d, ppm: 12.3 (C-2"'), 51.0 (C-1"'), 54.2 (C-2,6), 56.0 (CH3), 70.2 (CH2), 113.6 (C-2'), 114.9 (C-5'), 124.1 (C-6'), 128.2 (C-2",6”), 128.34 (C-1'), 128.4 (C-4"), 128.9 (C-3",5”), 132.5 (C-1”), 135.2 (C-3,5), $137.2(\mathrm{CH}), 149.2$ (C-4'), 149.3 (C-3'), and 186.9 (C-4) (Figure 12).

\section{2,6-bis-((E)-4-(benzyloxy)-3- methoxybenzylidene)-4-(tert- butyl)cyclohexan-1-one, 13}

Yield: (84\%); color: solid yellow; mp: $188-193{ }^{\circ} \mathrm{C}$; and molecular weight: 602.76 . FT-IR $\left(v, \mathrm{~cm}^{-1}\right): 3079$ (C ${ }_{s p}^{2}-\mathrm{H}$ str.), 2954 ( $\mathrm{C}_{s p}{ }^{3}-\mathrm{H}$ str.), 1598 (C=O str.), 1509 ( $\mathrm{C}=\mathrm{C}$ aromatic str.), 1433 ( $\mathrm{C}=\mathrm{C}$ alkenyl str.), and 1250 (C-O str.). ${ }^{1} \mathrm{H}-\mathrm{NMR}(500 \mathrm{MHz}, \mathrm{DMSO}-$ $\mathrm{d}_{6}$ ) d, ppm: 0.93 (s, H-2"', 9H), 1.38 (t, $J=12.0 \mathrm{~Hz}$,

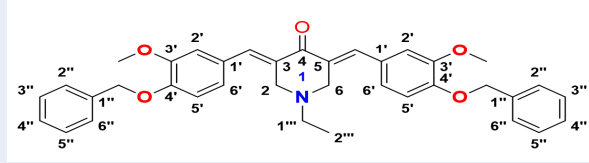

Figure 12: 3,5-bis-((E)-4-(benzyloxy)-3methoxybenzylidene)-1-ethylpiperidin-4-one,

12.

H-4, 1H), 3.10 (d, J=15.0 Hz, H-3, 5, 4H), 3.81 (s, $\left.\mathrm{CH}_{3}, 6 \mathrm{H}\right), 5.15$ (s, $\left.\mathrm{CH}_{2}, 4 \mathrm{H}\right), 7.14-7.46$ (m, Haromatic, $16 \mathrm{H})$, and $7.59\left(\mathrm{~s}, \mathrm{CH}_{2}, \mathrm{H}-5,2 \mathrm{H}\right) .{ }^{13} \mathrm{C}$ NMR (125 MHz, DMSO-d 6 ) d, ppm: 27.5 (C-2”'), 29.5 (C-3,5), 32.6 (C-1"'), 44.2 (C-4), 55.9 (CH3), 70.2 (CH2), 113.6 (C-2'), 114.7 (C-5'), 124.1 (C-6'), 128.3 (2",6"), 128.4 (C-1'), 128.8 (C-4"), 128.9 (C-3",5"), 134.7 (CH), 136.3 (C-1"), 137.2 (C-2,6), 148.9 (C-4'), 149.1 (C-3'), and 189.0 (C-1) (Figure 13).

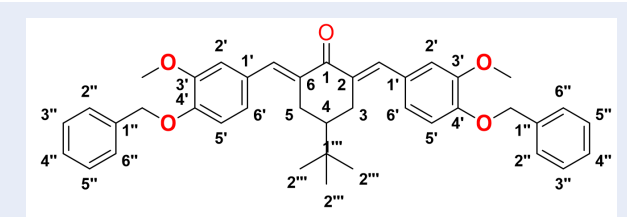

Figure 13: 2,6-bis-((E)-4-(benzyloxy)-3methoxybenzylidene)-4-(tert-butyl)cyclohexan1-one, 13.

\section{DISCUSSION}

\section{Molecular Docking}

The behavior of all 14 chalcone compounds were compared to that of tamoxifen. To be an effective drug, a compound must have optimum solubility of both water and fat, pass through the intestine, and be transported in aqueous blood before penetrating the cell membrane. Water solubility depends on the number of hydrogen bond donors relative to the compound's alkyl side chain. Low water solubility means slow absorption and action. Too many hydrogen bond donors contribute to low-fat solubility, leading to the drug's inability to cross the cell membrane. A simple method to evaluate the drug-like properties is to check the compliance with Lipinski's rule (rule of 5), which specifies the numbers of hydrophilic groups, molecular weight, and hydrophobicity. Lipinski's rule of five theorize that an active oral drug should have (i) not more than five hydrogen bond donors $(\mathrm{OH}$ and $\mathrm{NH}$ groups); (ii) not more than five hydrogen bond acceptors (notably $\mathrm{N}$ and $\mathrm{O}$ ); (iii) molecular weight 


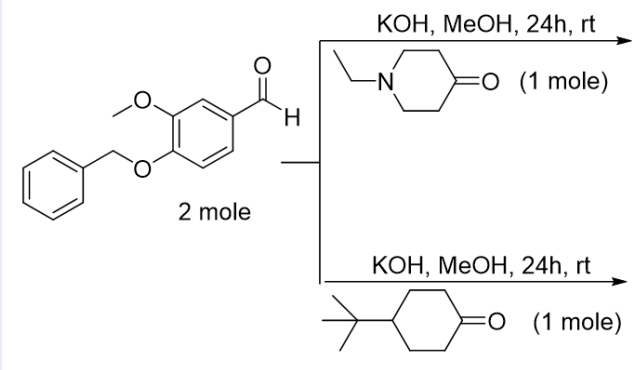

$12(78 \%)$

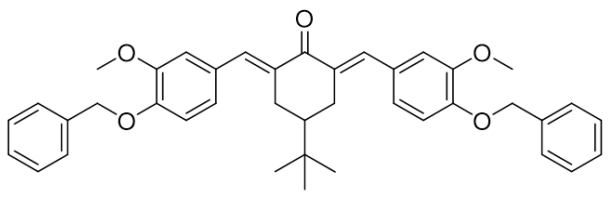

$13(84 \%)$

Scheme 4: Synthesis of bis-chalcones with methoxy and benzyloxy substituents, 12-13.

less than $500 \mathrm{~g} / \mathrm{mol}$; and (iv) octanol-water partition coefficient $(\log \mathrm{P})$ less than 5 . Interestingly, 8 out of the 11 thienyl chalcone derivatives complied with Lipinski's rule, as shown in Table 1. Moreover, Table 1 displays the computed scores of docking between the estrogen receptor alpha (ER $\alpha)$ structure (receptor) and all 11 synthesized compounds (ligands). The more negative the value, the higher the probability of an interaction between the ligand and the receptor. As expected, all of the 11 thienyl chalcone derivatives entered the ER $\alpha$ pocket as tamoxifen and possessed varying scores with the enclosed amino acids.

In comparison, the docking values of the bischalcone derivatives were more negative than tamoxifen derivatives in terms of predicting affinity to the receptor, except for compounds 11-13. At the same time, only 1 compound (6) is roughly applicable to the Lipinski rule, which has a Log P-value of 5.16 and a molecular weight less than $500 \mathrm{~g} / \mathrm{mol}$. These are considered to be very close to the Lipinski rule inquiry and possesses the activity of an in vitro assay against MCF-7 cancer cell more to a greater extent than tamoxifen, as presented in Table 1 .

The free binding energy of compound 6 was -10.32 $\mathrm{kcal} / \mathrm{mol}$, which was approximately the value of tamoxifen. Table 2 presents an overview of the predicted binding pose of compound 6 . It is noted from the 2D-molecular structure interaction of compound 6 in Figure 14, only one hydrogen bond with ARG394 and several hydrophobic interactions with leucine amino acid residues were established, making the interaction with the pocket as intense as it is in tamoxifen. It should be noted that the in vitro assay (MCF-7) of compound 6 was better than that of control (tamoxifen). This might be due to the existence of several interactions with amino acid (leucine), where inhibition of it plays a key role in reducing the activity of the estrogen receptor in breast cancer, as recently published $^{24}$.

\section{Cytotoxicity study}

The cytotoxicity activity of the 13 compounds was evaluated against human breast cancer cell line MCF7 at various concentrations, ranging from 1.563 to 25 $\mu \mathrm{M}$. The positive control, tamoxifen, was also investigated at the concentration of $3.25-50 \mu \mathrm{M}$. Compound 6 showed the most potent cytotoxicity with $\mathrm{IC}_{50}$ value of $4.4 \mu \mathrm{M}$ after incubation of MCF-7 cells with compound 6 for 48 hours.

Compounds 3, 5 and $\mathbf{9}$ showed moderate cytotoxicity activity with $\mathrm{IC}_{50}$ values of $35.5,19.3$ and $31.1 \mu \mathrm{M}$, respectively, while compounds $1,2,4,7,8,10,11,12$ and 13 were found to be inactive towards MCF-7. These results are summarized in Table 2 (the $\mathrm{IC}_{50}$ value of tamoxifen is shown as $17.9 \mu \mathrm{M}$ ). The results revealed that compound $\mathbf{6}$ showed more potent cytotoxicity compared to the reference anticancer drug, tamoxifen. Since compound 6 showed an $\mathrm{IC}_{50}$ value below $10 \mu \mathrm{M}$ against MCF-7 breast cancer cells, it could potentially be an effective drug for treatment of breast cancer.

\section{CONCLUSION}

A series of thertine bis-chalcones were successfully synthesized between terephthalaldehyde with 3-acetyl-5-chlorothiophene (1), between terephthalaldehyde with 3-acetyl-2,5-dichlorothiophene (2), between terephthalaldehyde with 3-acetyl-2,5dichlorothiophene and 3-acetyl- 5-chlorothiophene (3), and between cyclic ketone derivatives with phenyl aldehyde derivatives (4-13). All the compounds were characterized using FTIR, ${ }^{1} \mathrm{H}$ and ${ }^{13} \mathrm{C}$ 
Table 1: Chemical properties based on Lipinski's rule ofall bis-chalcone derivatives (1-13)and the tamoxifen (control)

\begin{tabular}{|c|c|c|c|c|c|c|}
\hline $\begin{array}{l}\text { Com- } \\
\text { pound }\end{array}$ & $\begin{array}{l}\text { M.W } \\
\text { (g/mol) }\end{array}$ & $\begin{array}{c}\log \\
P\end{array}$ & $\begin{array}{l}\text { H-bond } \\
\text { donor }\end{array}$ & $\begin{array}{l}\text { H-bond } \\
\text { acceptor }\end{array}$ & $\begin{array}{c}\text { FBE } \\
(\text { Kcal } / \mathrm{mol})\end{array}$ & $\begin{array}{l}\text { Inhibition Constant } \mathrm{Ki} \text {, } \\
\qquad(\mathrm{mM})\end{array}$ \\
\hline 151 & 419.34 & 5.85 & 1 & 0 & -10.18 & 0.034 \\
\hline 162 & 453.79 & 6.16 & 0 & 0 & -9.14 & 0.200 \\
\hline 173 & 488.23 & 6.48 & 0 & 0 & -9.81 & 0.064 \\
\hline 184 & 365.20 & 5.65 & 1 & 0 & -8.78 & 0.369 \\
\hline 195 & 379.23 & 6.07 & 0 & 0 & -10.02 & 0.045 \\
\hline 206 & 407.07 & 5.16 & 1 & 0 & -10.32 & 0.031 \\
\hline 217 & 435.33 & 7.62 & 0 & 0 & -8.51 & 0.575 \\
\hline 228 & 506.59 & 6.98 & 0 & 0 & -9.26 & 0.164 \\
\hline 239 & 532.63 & 7.18 & 0 & 0 & -9.42 & 0.124 \\
\hline 2410 & 575.69 & 6.68 & 0 & 0 & -9.29 & 0.154 \\
\hline 2511 & 602.76 & 9.15 & 1 & 0 & -6.56 & 15.61 \\
\hline 2612 & 575.69 & 6.68 & 0 & 0 & -6.47 & 18.02 \\
\hline 2713 & 602.76 & 9.15 & 0 & 0 & -6.05 & 36.57 \\
\hline $\begin{array}{l}\text { Tamox- } \\
\text { ifen }\end{array}$ & 371.51 & 6.07 & 0 & 2 & -10.40 & 0.027 \\
\hline
\end{tabular}

Table 2: Compounds 1-11 and Tamoxifen IC $\mathrm{IC}_{50}$ value $(\mu \mathrm{M})$ against MCF-7 cell line

\begin{tabular}{lc}
\hline Compound & IC $_{50}$ \\
\hline Tamoxifen $(\mathrm{MW}=371.51)$ & $17.9 \pm 1.2$ \\
$1($ Yield $=68 \%, \mathrm{MW}=419.34)$ & $\mathrm{NA}$ \\
$2($ Yield $=71 \%, \mathrm{MW}=453.79)$ & $\mathrm{NA}$ \\
$3($ Yield $=74 \%, \mathrm{MW}=488.23)$ & $35.5 \pm 3.8$ \\
$4($ Yield $=85 \%, \mathrm{MW}=365.20)$ & $\mathrm{NA}$ \\
$5($ Yield $=79 \%, \mathrm{MW}=379.23)$ & $19.3 \pm 0.7$ \\
$6($ Yield $=84 \%, \mathrm{MW}=407.07)$ & $4.4 \pm 0.1$ \\
$7($ Yield $=79 \%, \mathrm{MW}=435.33)$ & $\mathrm{NA}$ \\
$8($ Yield $=78 \%, \mathrm{MW}=506.59)$ & $\mathrm{NA}$ \\
$9($ Yield $=75 \%, \mathrm{MW}=532.63)$ & $\mathrm{NA}$ \\
$10($ Yield $=78 \%, \mathrm{MW}=575.69)$ & $\mathrm{NA}$ \\
$11($ Yield $=69 \%, \mathrm{MW}=602.76)$ & $\mathrm{NA}$ \\
$12($ Yield $=78 \%, \mathrm{MW}=575.69)$ & $\mathrm{NA}$ \\
$13($ Yield $=84 \%, \mathrm{MW}=602.76)$ & $31.1 \pm 1.3$ \\
\hline
\end{tabular}




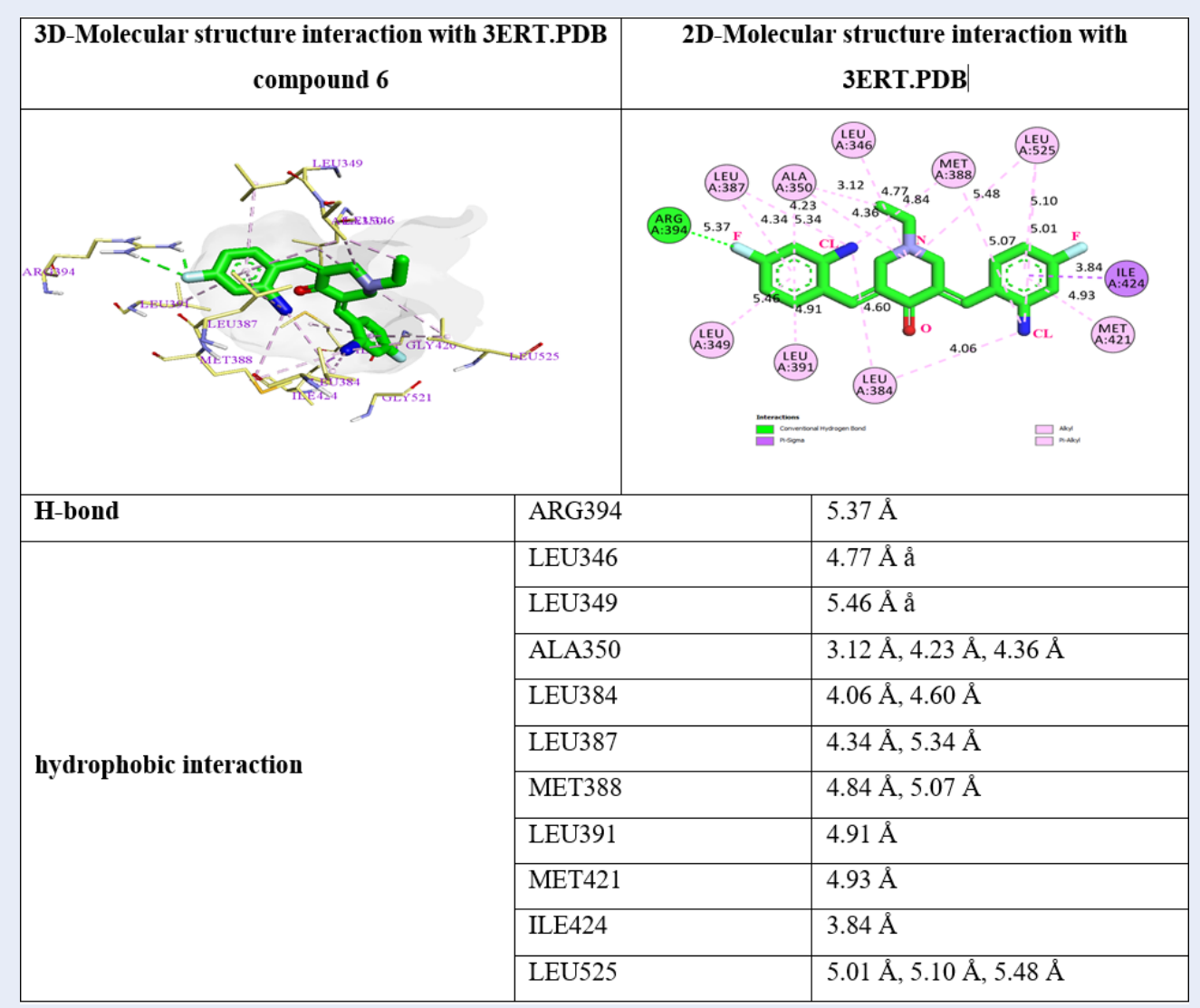

Figure 14: Summary of the best predicted binding poses of compound 6 . In the scaffold, green color represents the carbon atoms, red for oxygen, sky blue for fluorine, dark blue for chlorine, and pale blue for the nitrogen atom.

NMR spectroscopy. The cytotoxic activity of all the synthesized compounds was evaluated against MCF-7 breast cancer cell line. Compounds 5 and $\mathbf{6}$ were found to have $\mathrm{IC}_{50}$ values about $20 \mu \mathrm{M}$. These compounds are good candidates to be selected for further studies to develop anticancer drugs. The structure-activity relationship studies showed that bis-chalcone compounds with substituted chlorine and fluorine atoms at the ortho-chlorine and parafluorine position of their aromatic rings exhibited greater cytotoxic activity against MCF-7 cell line compared with other compounds. Moreover, in the cytotoxic activity tests on MCF-7 breast cancer cells, the bis-chalcones derivatives from cyclohexane, 1-ethylpiperidin-4-one were found to be more potent than bis-chalcone derivatives from acetone, cyclopentanone, 4-(tert-butyl)cyclohexan-1-one and terephthalaldehyde.

\section{ABBREVIATIONS}

None.

\section{ACKNOWLEDGMENTS}

The authors would like to thank Universiti Sains Malaysia, Penang, Malaysia and the Malaysian Government for the Fundamental Research Grant Scheme (FRGS) 1/2019 (203.PKIMIA.6711789) which was used to finance this research work.

\section{AUTHOR'S CONTRIBUTIONS}

Conceptualization and planning, M.K.;Docking studies, synthesis and characterization, M.M.A., M.K.; Cytotoxity work, S.M.S and M.A.; Supervision, M.K.; writing-original draft, M.M.A., M.K.; writing-review and editing, M.K., M.M.A. All authors read and approved the final manuscript.

\section{FUNDING}

None. 


\section{AVAILABILITY OF DATA AND MATERIALS}

Data and materials used and/or analyzed during the current study are available from the corresponding author on reasonable request.

\section{ETHICS APPROVAL AND CONSENT TO PARTICIPATE}

Not applicable.

\section{CONSENT FOR PUBLICATION}

Not applicable.

\section{COMPETING INTERESTS}

The authors declare that they have no competing interests.

\section{REFERENCES}

1. Ferrando AA, Neuberg DS, Staunton J, Loh ML, Huard C, Raimondi SC. Gene expression signatures define novel oncogenic pathways in T cell acute lymphoblastic leukemia. Cancer Cell. 2002;1(1):75-87. PMID: 12086890. Available from: 10.1016/S1535-6108(02)00018-1.

2. Jiang WG, Sanders AJ, Katoh $M$, Ungefroren $H$, Gieseler $F$, Prince $M$. Tissue invasion and metastasis: Molecular, biological and clinical perspectives. Semin Cancer Biol. 2015;35:24475. PMID: 25865774. Available from: 10.1016/j.semcancer. 2015.03.008.

3. Devesa SS, Bray F, Vizcaino AP, Parkin DM. International lung cancer trends by histologic type: male:female differences diminishing and adenocarcinoma rates rising. Int J Cancer. 2005;117(2):294-9. PMID: 15900604. Available from: 10.1002/ ijc. 21183 .

4. Sharma GN, Dave R, Sanadya J, Sharma P, Sharma KK. Various types and management of breast cancer: an overview. J Adv Pharm Technol Res. 2010;1 (2):109-26. PMID: 22247839.

5. Chen Q, Xu L, Liang C, Wang C, Peng R, Liu Z. Photothermal therapy with immune-adjuvant nanoparticles together with checkpoint blockade for effective cancer immunotherapy. Nat Commun. 2016;7(1):13193. PMID: 27767031. Available from: 10.1038/ncomms13193.

6. Adam JK, Odhav B, Bhoola KD. Immune responses in cancer Pharmacol Ther. 2003;99(1):113-32. PMID: 12804702. Available from: 10.1016/S0163-7258(03)00056-1.

7. Stewart TJ, Smyth MJ. Improving cancer immunotherapy by targeting tumor-induced immune suppression. Cancer Metastasis Rev. 2011;30(1):125-40. PMID: 21249424. Available from: 10.1007/s10555-011-9280-5.

8. Burkholder B, Huang RY, Burgess R, Luo S, Jones VS, Zhang W. Tumor-induced perturbations of cytokines and immune cell networks. Biochim Biophys Acta. 2014;1845(2):182-201. PMID: 24440852.

9. Kidd PM. The use of mushroom glucans and proteoglycans in cancer treatment. Altern Med Rev. 2000;5(1):4-27. PMID: 10696116.
10. Feng SS, Chien S. Chemotherapeutic engineering: application and further development of chemical engineering principles for chemotherapy of cancer and other diseases. Chem Eng Sci. 2003;58(18):4087-114. Available from: 10.1016/S00092509(03)00234-3.

11. Geerts WH, Pineo GF, Heit JA, Bergqvist D, Lassen MR, Colwell CW. Prevention of venous thromboembolism: the Seventh ACCP Conference on Antithrombotic and Thrombolytic Therapy. Chest. 2004;126(3):338-400. PMID: 15383478. Available from: 10.1378/chest.126.3_suppl.338S.

12. Ji X, Lu Y, Tian H, Meng X, Wei M, Cho WC. Chemoresistance mechanisms of breast cancer and their countermeasures. Biomed Pharmacother. 2019;114:108800. PMID: 30921705. Available from: 10.1016/j.biopha.2019.108800.

13. Rammohan A, Reddy JS, Sravya G, Rao CN, Zyryanov GV. Chalcone synthesis, properties and medicinal applications: a review. Environ Chem Lett. 2020;18(2):1-26. Available from: 10.1007/s10311-019-00959-w.

14. Mathew B, Suresh J, Anbazghagan S, Paulraj J, Krishnan GK. Heteroaryl chalcones: mini review about their therapeutic voyage. Biomedicine \{\&amp;\} Preventive Nutrition. 2014;4(3):451-8. Available from: 10.1016/j.bionut.2014.04.003.

15. Asiri AM, Marwani HM, Alamry KA, Al-Amoudi MS, Khan SA, ElDaly SA. Green synthesis, characterization, photophysical and electrochemical properties of bis-chalcones. Int J Electrochem Sci. 2014;9:799-809.

16. Alakurtti S, Mäkelä T, Koskimies S, Yli-Kauhaluoma J. Pharmacological properties of the ubiquitous natural product betulin. Eur J Pharm Sci. 2006;29(1):1-13. PMID: 16716572. Available from: 10.1016/j.ejps.2006.04.006.

17. Grunberger D, Banerjee R, Eisinger K, Oltz EM, Efros L, Caldwell M. Preferential cytotoxicity on tumor cells by caffeic acid phenethyl ester isolated from propolis. Experientia. 1988;44(3):230-2. PMID: 2450776. Available from: 10.1007/ BF01941717.

18. Baba M, Ohmura M, Kishi N, Okada $Y$, Shibata S, Peng J. Saponins isolated from Allium chinense G. Don and antitumor-promoting activities of isoliquiritigenin and laxogenin from the same drug. Biol Pharm Bull. 2000;23(5):660-2. PMID: 10823685. Available from: 10.1248/bpb.23.660.

19. Edwards ML, Stemerick DM, Sunkara PS. Chalcones: a new class of antimitotic agents. J Med Chem. 1990;33(7):1948-54. PMID: 2362275. Available from: 10.1021/jm00169a021.

20. Torigoe T, Arisawa M, Itoh S, Fujiu M, Maruyama HB. Antimutagenic chalcones: antagonizing the mutagenicity of benzo(a)pyrene on Salmonella typhimurium. Biochem Biophys Res Commun. 1983;112(3):833-42. PMID: 6303339. Available from: 10.1016/0006-291X(83)91693-5.

21. Shiau AK, Barstad D, Loria PM, Cheng L, Kushner PJ, Agard DA. The structural basis of estrogen receptor/coactivator recognition and the antagonism of this interaction by tamoxifen. Cell. 1998;95(7):927-37. PMID: 9875847. Available from: 10.1016/S0092-8674(00)81717- 1 .

22. Edenharder R, von Petersdorff I, Rauscher R. Antimutagenic effects of flavonoids, chalcones and structurally related compounds on the activity of 2-amino-3-methylimidazo[4,5f] quinoline (IQ) and other heterocyclic amine mutagens from cooked food. Mutat Res. 1993;287(2):261-74. PMID: 7685486. Available from: 10.1016/0027-5107(93)90019-C.

23. Aljamali N, Daylee SH, Kadhium AJ. Review on ChemicalBiological Fields of Chalcone Compounds. Forefront Journal of Engineering \{\&amp;\}Technology. 2020;2(1):33-44.

24. Saito Y, Li L, Coyaud E, Luna A, Sander C, Raught B. LLGL2 rescues nutrient stress by promoting leucine uptake in $E R+$ breast cancer. Nature. 2019;569(7755):275-9. PMID: 30996345. Available from: 10.1038/s41586-019-1126-2. 